\title{
Duloxetine treatment adherence across mental health and chronic pain conditions
}

This article was published in the following Dove Press journal:

ClinicoEconomics and Outcomes Research

II February 2014

Number of times this article has been viewed

\author{
Stephen L Able' \\ Zhanglin $\mathrm{Cui}^{2}$ \\ Wei Shen ${ }^{2}$ \\ 'Global Health Outcomes, Eli Lilly \\ and Company, Indianapolis, IN, USA; \\ ${ }^{2}$ Global Statistical Sciences, Eli Lilly \\ and Company, Indianapolis, IN, USA
}

Purpose: This study applied a uniform methodology for measuring and comparing duloxetine adherence in the treatment of multiple chronic medical conditions.

Materials and methods: Study patients 18-64 years of age initiating duloxetine therapy during 2008 were identified from a large managed care database. The study was restricted to patients with continuous health plan eligibility for 12 months pre- and post-duloxetine initiation. Study patients had $\geq 1$ medical claim with an inpatient or outpatient diagnosis of one (and only one) of the following conditions: major depressive disorder (MDD); generalized anxiety disorder (GAD); fibromyalgia, diabetic peripheral neuropathic pain; or chronic musculoskeletal pain, as established in studies in patients with osteoarthritis and chronic lower back pain (CLBP). Patients initiating duloxetine who had two or more of the six studied conditions were not included in this study, thereby avoiding the need to differentiate between primary and secondary diagnoses from the claims records. Adherence rate was defined as the percentage of patients with a 365-day medication possession ratio $\geq 0.8$.

Results: A total of 20,490 patients initiated duloxetine treatment during 2008 with a diagnosis of one of the studied conditions during the study period. The adherence rate in our sample was $34.6 \%$ and was highest among patients with MDD (37.3\%) and lowest for patients with CLBP (29.9\%). In general, adherence among patients with MDD and GAD was greater than among those with a chronic pain condition.

Conclusion: Adherence among newly initiated duloxetine patients varied modestly across the medical conditions for which it was used. After adjusting for potential confounders, differences between the mental conditions (MDD and GAD) and the chronic pain conditions (CLBP, osteoarthritis, and diabetic peripheral neuropathic pain) were statistically significant. These results may be useful in the determination of expectations of adherence, and how it may differ for each of the conditions studied.

Keywords: adherence, duloxetine, major depressive disorder, generalized anxiety disorder, chronic lower back pain, diabetic peripheral neuropathic pain

\section{Introduction}

Suboptimal treatment adherence is widely viewed as a pervasive impediment to the successful treatment of chronic illness. Studies have consistently shown that lower adherence is associated with diminished efficacy and increased health care costs. ${ }^{1-6}$ Health insurance plans have long shown interest in the measurement and monitoring of adherence to medications included in their formularies, and will likely continue to do so as they attempt to develop and incorporate strategies designed to increase adherence into their health care quality improvement efforts. ${ }^{7}$
Correspondence: Stephen L Able Eli Lilly and Company, I Lilly Corporate Center, Indianapolis, IN 46285-000I, USA

Tel +13172763612

Fax + I 3172777444

Email able_stephen_ı@lilly.com 
While most medications are used primarily in the treatment of a single or small number of closely related diseases, some, like those in the antidepressant class, are used in more than one disease state. Most published studies addressing the quantitative measurement of adherence focus on one or more drugs or drug classes in the treatment of a single medical condition. In fact, we know of but two studies published within the past 25 years that attempted direct comparisons of adherence across multiple chronic medical conditions. ${ }^{8,9}$ Both reported variable, but uniformly suboptimal, medication adherence across the chronic disease states included in their studies. Neither, however, addressed the use of a single pharmaceutical agent across a range of medical conditions, nor included antidepressants among the agents studied.

Duloxetine, a dual serotonin (5-hydroxytryptomine) and norepinephrine reuptake inhibitor, ${ }^{10}$ considered a member of the antidepressant class of medications, has been shown to be efficacious in several mental and chronic pain conditions: major depressive disorder (MDD); ${ }^{11}$ generalized anxiety disorder (GAD); ${ }^{12}$ fibromyalgia (FM); ${ }^{13}$ diabetic peripheral neuropathic pain (DPNP); ${ }^{14}$ and chronic musculoskeletal pain, as established in studies in patients with osteoarthritis (OA) ${ }^{15}$ and chronic lower back pain (CLBP). ${ }^{16}$ Claims-based retrospective studies of duloxetine adherence and/or persistence have been conducted separately in MDD, ${ }^{6,17,18} \mathrm{FM},{ }^{5,19}$ and DPNP, ${ }^{4,20}$ but none were designed to provide a direct comparison across multiple disease states within a uniform analytical framework. Statistically meaningful comparisons across the individual studies are hampered by the use of different data sources and study periods, variations in analytical methods, and a variety of technical assumptions made in the calculation algorithms used to estimate adherence rates.

The objective of the present study was to apply a single, uniform methodology for measuring and comparing rates of duloxetine treatment adherence across a range of chronic medical conditions, for which it has demonstrated efficacy, with particular attention paid to whether or not, and by how much, adherence varies across mental and chronic pain conditions.

\section{Materials and methods}

\section{Data source and patient selection criteria}

Data utilized in this study were drawn from a large national managed care database (Thomson Reuters MarketScan ${ }^{\circledR}$ Commercial Claims and Encounters Database; Thomson Reuters, New York City, NY, USA) for 2007-2009. The database provides access to integrated inpatient, outpatient, and pharmacy claims data, as well as demographics (for example, age, geographic region of residence), health insurance plan type, and enrollment status (beginning and ending dates of health insurance plan enrollment and, hence, plan eligibility) on employees and their dependants from more than 100 large employer-sponsored health insurance plans in the United States. Medical records included dates of service and International Classification of Diseases, 9th Revision, Clinical Modification (ICD-9-CM) diagnosis and procedure codes. Pharmacy records provided the National Drug Code, dispense date, and quantity dispensed for each duloxetine prescription filled during the study period. Patients included in the study were aged 18-64 years who initiated duloxetine therapy between January 1, 2008 and December 31, 2008. The date of the first filled prescription for duloxetine during the study period for which there was no duloxetine pill coverage during the previous 90 days (look-back period) was defined as the index date. The study was restricted to patients who were continuously enrolled in their health insurance plan from 12 months before to 12 months after that index date.

Study patients were required to have at least one medical claim with an associated inpatient or outpatient diagnosis of one (and only one) of the following medical conditions (defined in terms of ICD-9-CM codes, listed in parentheses): MDD (296.2, 296.3); GAD (300.02); FM (729.1), DPNP (250.6, 357.2); OA (715.xx); and CLBP (722.10, 722.83, 722.93, $724.02,724.2,724.3,724.5,724.8)$. Patients initiating duloxetine who had two or more of the six studied conditions were not included in this study, thereby avoiding the need to differentiate between primary and secondary diagnoses from the claims records. The qualifying diagnosis had to be between 3 months prior and 1 month following the index date. Because ICD-9-CM codes for lower back pain do not distinguish between chronic and acute conditions, patients were required to have received care coded with at least two lower back pain diagnosis codes more than 90 days apart to qualify for inclusion in the CLBP study cohort. ${ }^{21}$ Because an initial diagnosis of OA is frequently replaced with a different diagnosis code in subsequent patient visits, inclusion in the OA cohort required a second OA diagnoses within 1 year (preceding or following) the index date.

\section{Adherence}

There are a variety of definitions and algorithms available for use in retrospective studies of adherence. ${ }^{22,23} \mathrm{We}$ defined adherence on the basis of the medication possession ratio (MPR) for each patient. ${ }^{24}$ More specifically, we calculated MPR as the total number of days of duloxetine therapy dispensed during the 12 months after the index date, 
divided by $365.23,25$ The adherence rate was defined as the percentage of patients with an MPR $\geq 0.8$.

\section{Statistical analysis}

Descriptive statistics were used to summarize patient demographic characteristics, MPR, and adherence rates. Pairwise comparison cohorts were examined via Student's $t$-tests for MPR (a continuous variable); chi-square tests were used for categorical adherence rate estimates.

We also conducted stepwise multiple logistic regression analysis to allow us to re-examine the statistical significance of observed adherence rates after adjusting for potentially confounding factors, which were suggested in previous studies of duloxetine treatment adherence in MDD, FM, and DPNP. ${ }^{4-6,17-20}$ A model was specified for adherence versus nonadherence, as defined above, and included an indicator for the condition treated, as referenced against MDD. Variables suggested in previous research for individual medical conditions were incorporated into the model: demographics (patient age, sex, geographic region of residence, and health insurance plan type); comorbid health conditions; pre-index date medication history; and initial dosing levels. Also included at entry to the stepwise multiple logistic regression analysis were all 17 ICD-9-CM classes of body system disorders; 27 selected individual diseases or disease categories that are associated with depression, chronic pain, safety, or efficacy of treatment; 17 individual medications or medication categories, for which prescriptions were dispensed within the 12 months prior to index; and treatment dose, which was grouped as low (subtherapeutic dose: $<60 \mathrm{mg} /$ day), medium (recommended dose: $60 \mathrm{mg} /$ day), and high (above the recommended dose: $>60 \mathrm{mg} /$ day), as given on the duloxetine product label. ${ }^{26}$

Data processing and statistical analyses were conducted with SAS version 9.2 (SAS Institute Inc., Cary, NC, USA). Statistical significance testing of adherence rates was conducted at the 0.05 level, while the stepwise logistic regressions were conducted with 0.1 as the entry and 0.05 as the exit significance levels.

\section{Results}

Of a total of 175,509 patients in the managed care database who initiated duloxetine treatment between January 1, 2008 and December 31, 2008, 48,280 met the continuous enrollment criteria. Of these, a total of 20,490 recorded diagnoses within 90 days before and 30 days after the study index date for only one of the studied conditions during the designated diagnosis period, which were distributed across the study cohorts as follows: MDD (number $[\mathrm{n}]=8,334$ ); GAD $(n=1,477)$; FM $(n=3,630)$; DPNP $(n=607)$; OA $(n=1,458)$; and CLBP $(n=4,984)$.

Patient demographic characteristics for the study sample are reported in Table 1. Most of our sample was composed of women $(74.3 \%)$. The largest proportion of women was found among duloxetine initiators diagnosed with FM (90.3\%), the smallest among those with DPNP (55.7\%). The average age of our sample was 46.3 years. Individuals with OA ( mean $=53.4$ years $)$ and DPNP (mean $=53.1$ years $)$ formed the oldest groups, while those with GAD (mean $=41.5$ years) and MDD (mean $=44.2$ years) formed the youngest. Preferred provider organizations were the most common health insurance plan type in our sample, with no notable variations across study cohorts. The database used in this study is dominated by subjects from the south (and, to a lesser extent, the north central) area of the United States, and this is reflected in our sample.

Adherence rates for each of the disease states are presented in Table 2. In our sample, the average MPR was 0.54 and the rate of adherence was $34.6 \%$. Pairwise statistical tests showed that the adherence rate among patients with MDD $(37.3 \%)$ was significantly higher $(P<0.05)$ than for any other patient cohort, except OA (35.7\%). The adherence rate among patients with CLBP (29.9\%) was significantly lower $(P<0.05)$ than for any other cohort, with the exception of DPNP (31.0\%). The adherence rate for patients with DPNP was significantly lower $(P<0.05)$ than for any other cohort, except CLBP and GAD (33.5\%).

The results of the stepwise logistic regression, including odds ratios and confidence intervals, are presented in Tables 3 and 4. Regression results showed that patients with MDD were significantly more likely to be adherent than patients with DPNP, OA, and CLBP. Females were significantly more likely to be adherent to duloxetine treatment than were men, as were older patients (relative to those 18-25 years old). Health insurance plan type did not have a significant effect on adherence rates, while geographical differences were limited to a somewhat greater likelihood of adherence by patients from the north central United States versus those from the south. Patients initiating on duloxetine treatment at the recommended level of $60 \mathrm{mg}$ /day were more likely to be adherent than those initiating at doses greater than or less than $60 \mathrm{mg} /$ day.

A number of variables related to patient medical history (comorbidities, body system disorders, and prior medication use) were also identified by the logistic regression as 
Table I Patient demographics and dosing, by patient cohort

\begin{tabular}{|c|c|c|c|c|c|c|c|}
\hline & $\begin{array}{l}\text { MDD } \\
(n=8,334)\end{array}$ & $\begin{array}{l}\text { GAD } \\
(n=1,477)\end{array}$ & $\begin{array}{l}\text { FM } \\
(n=3,630)\end{array}$ & $\begin{array}{l}\text { DPNP } \\
(n=607)\end{array}$ & $\begin{array}{l}\text { OA } \\
(n=1,458)\end{array}$ & $\begin{array}{l}\text { CLBP } \\
(n=4,984)\end{array}$ & $\begin{array}{l}\text { Total } \\
(\mathrm{N}=20,490)\end{array}$ \\
\hline \multicolumn{8}{|l|}{$\operatorname{Sex}(\%)$} \\
\hline Male & 27.4 & 32.0 & 9.7 & 44.3 & 23.4 & 31.0 & 25.7 \\
\hline Female & 72.6 & 68.0 & 90.3 & 55.7 & 76.6 & 69.0 & 74.3 \\
\hline \multicolumn{8}{|l|}{ Age, years (\%) } \\
\hline $18-25$ & 7.8 & 8.7 & 2.0 & 0.0 & 0.3 & 2.0 & 4.7 \\
\hline $26-35$ & 11.7 & 17.5 & 9.5 & 2.5 & 1.7 & 8.3 & 9.9 \\
\hline $36-45$ & 23.9 & 27.7 & 23.2 & 8.6 & 8.9 & 22.2 & 22.1 \\
\hline $46-55$ & 33.3 & 30.5 & 37.4 & 34.9 & 34.0 & 37.2 & 34.9 \\
\hline $56-64$ & 23.3 & 15.6 & 28.0 & 54.0 & 55.1 & 30.3 & 28.5 \\
\hline Mean $( \pm S D)$ & $44.2(11.7)$ & $4 \mid .5(\mid 1.5)$ & $47.1(9.8)$ & $53.1(7.3)$ & $53.4(7.1)$ & $47.7(9.9)$ & $46.3(10.5)$ \\
\hline \multicolumn{8}{|c|}{ Health insurance plan type (\%) } \\
\hline PPO & 67.3 & 71.1 & 68.4 & 66.2 & 68.5 & 69.8 & 68.4 \\
\hline HMO & 15.7 & 11.7 & 12.6 & 12.5 & 12.8 & 11.6 & 13.6 \\
\hline POS & 10.5 & 10.8 & 10.7 & 11.9 & 9.9 & 10.4 & 10.5 \\
\hline Comprehensive & 2.4 & 2.0 & 2.8 & 4.0 & 4.7 & 3.2 & 2.8 \\
\hline CDHP & 1.6 & 1.8 & 2.1 & 1.8 & 1.0 & 1.9 & 1.7 \\
\hline \multicolumn{8}{|c|}{ United States geographic region of residence (\%) } \\
\hline South & 40.5 & 50.7 & 48.9 & 56.0 & 51.3 & 51.4 & 46.6 \\
\hline North central & 33.5 & 31.4 & 30.3 & 24.2 & 30.3 & 29.0 & 31.2 \\
\hline West & 18.0 & 8.9 & 14.6 & 13.2 & 11.9 & 13.4 & 15.0 \\
\hline Northeast & 7.6 & 8.7 & 6.0 & 6.3 & 6.0 & 5.7 & 6.8 \\
\hline Unknown & 0.4 & 0.3 & 0.3 & 0.3 & 0.5 & 0.5 & 0.5 \\
\hline \multicolumn{8}{|l|}{ Initial dose (\%) } \\
\hline 60 mg/day & 58.2 & 58.4 & 60.4 & 62.6 & 62.2 & 61.1 & 59.7 \\
\hline$<60 \mathrm{mg} /$ day & 30.1 & 35.9 & 33.8 & 30.5 & 30.9 & 32.2 & 31.7 \\
\hline$>60 \mathrm{mg} / \mathrm{day}$ & 11.7 & 5.7 & 5.8 & 6.9 & 6.9 & 6.7 & 8.5 \\
\hline
\end{tabular}

Abbreviations: MDD, major depressive disorder; n, number; GAD, generalized anxiety disorder; FM, fibromyalgia; DPNP, diabetic peripheral neuropathic pain; OA, osteoarthritis; CLBP, chronic lower back pain; N, total number of patients; SD, standard deviation; PPO, preferred provider organization; HMO, health maintenance organization; POS, point of service; CDHP, consumer-driven health plan.

affecting observed adherence rates. Infections, insomnia, miscellaneous pain conditions, and psychiatric disorders (other than MDD and GAD) were related to lower adherence to duloxetine treatment, while nerve conditions, dyslipidemia, sleep apnea and OA (prior to the look-back period) were positively related. Prior use of benzodiazepines, duloxetine (pre-washout), muscle relaxants, and opioids was related to lower adherence, while prior use of nonduloxetine serotonin and norepinephrine reuptake inhibitors and selective serotonin reuptake inhibitors (SSRIs) was related to higher adherence.

\section{Discussion}

The purpose of this study of 20,490 commercially-insured patients initiating treatment with duloxetine during 2008 was to calculate and compare adherence rates for a single medication across multiple medical conditions using a single data source, over identical study periods, with a uniform set

Table 2 Medication possession ratio and adherence rates (365 days) by patient cohort, as well as pairwise Student's t-tests

\begin{tabular}{|c|c|c|c|c|c|c|c|}
\hline & $\begin{array}{l}\text { MDD } \\
(n=8,334)\end{array}$ & $\begin{array}{l}\text { GAD } \\
(n=1,477)\end{array}$ & $\begin{array}{l}\text { FM } \\
(n=3,630)\end{array}$ & $\begin{array}{l}\text { DPNP } \\
(n=607)\end{array}$ & $\begin{array}{l}\text { OA } \\
(n=1,458)\end{array}$ & $\begin{array}{l}\text { CLBP } \\
(n=4,984)\end{array}$ & $\begin{array}{l}\text { Total } \\
(N=20,490)\end{array}$ \\
\hline MPR $( \pm S D)$ & $0.56(0.35)$ & $0.54(0.35)$ & $0.54(0.35)$ & $0.52(0.35)$ & $0.56(0.35)$ & $0.50(0.35)$ & $0.54(0.35)$ \\
\hline Adherence rate, \% & 37.3 & 33.5 & 35.3 & 31.0 & 35.7 & 29.9 & 34.6 \\
\hline MDD & - & $P<0.05$ & $P<0.05$ & $P<0.05$ & NS & $P<0.05$ & \\
\hline GAD & - & - & NS & NS & NS & $\mathrm{P}<0.05$ & \\
\hline $\mathrm{FM}$ & - & - & - & $P<0.05$ & NS & $P<0.05$ & \\
\hline DPNP & - & - & - & - & $P<0.05$ & NS & \\
\hline $\mathrm{OA}$ & - & - & - & - & - & $P<0.05$ & \\
\hline
\end{tabular}

Abbreviations: MDD, major depressive disorder; n, number; GAD, generalized anxiety disorder; FM, fibromyalgia; DPNP, diabetic peripheral neuropathic pain; OA, osteoarthritis; CLBP, chronic lower back pain; N, total number of patients; MPR, medication possession ratio; SD, standard deviation; NS, not significant. 
Table 3 Odds ratios and $95 \% \mathrm{Cls}$ for stepwise logistic regression of adherence (part I)

\begin{tabular}{|c|c|c|}
\hline & \multicolumn{2}{|l|}{ Adherence } \\
\hline & Odds ratio & $95 \% \mathrm{Cl}$ \\
\hline \multicolumn{3}{|c|}{ Treated condition (ref: MDD) } \\
\hline GAD & 1.03 & $0.89-1.21$ \\
\hline FM & 0.99 & $0.87-1.13$ \\
\hline DPNP & 0.70 & $0.57-0.86$ \\
\hline OA & 0.80 & $0.67-0.96$ \\
\hline CLBP & 0.81 & $0.72-0.92$ \\
\hline \multicolumn{3}{|l|}{ Sex (ref: male) } \\
\hline Female & 1.16 & $1.09-1.25$ \\
\hline \multicolumn{3}{|l|}{ Age, years (ref: 18-25) } \\
\hline $26-35$ & 1.24 & $1.03-1.50$ \\
\hline $36-45$ & 1.80 & $1.52-2.13$ \\
\hline $46-55$ & 2.15 & $1.82-2.54$ \\
\hline $56-64$ & 2.64 & $2.23-3.13$ \\
\hline \multicolumn{3}{|c|}{ Health insurance plan type (ref: comprehensive) } \\
\hline HMO & 0.85 & $0.70-1.03$ \\
\hline POS & 0.82 & $0.68-1.00$ \\
\hline POS with capitation & 1.13 & $0.63-2.03$ \\
\hline PPO & 1.01 & $0.84-1.19$ \\
\hline \multicolumn{3}{|c|}{ United States geographic region of residence (ref: South) } \\
\hline North central & 1.21 & $1.13-1.30$ \\
\hline Northeast & I.II & $0.98-1.26$ \\
\hline West & 1.02 & $0.93-1.12$ \\
\hline Unknown & 0.89 & $0.54-1.47$ \\
\hline \multicolumn{3}{|c|}{ Initial dose (ref: $60 \mathrm{mg} /$ day) } \\
\hline$<60 \mathrm{mg} /$ day & 0.75 & $0.70-0.80$ \\
\hline$>60 \mathrm{mg} / \mathrm{day}$ & 0.81 & $0.72-0.90$ \\
\hline
\end{tabular}

Abbreviations: $\mathrm{Cl}$, confidence interval; ref, reference; MDD, major depressive disorder; GAD, generalized anxiety disorder; FM, fibromyalgia; DPNP, diabetic peripheral neuropathic pain; OA, osteoarthritis; CLBP, chronic lower back pain; HMO, health maintenance organization; POS, point of service; PPO, preferred provider organization.

of technical definitions and assumptions in its calculation algorithms. To our knowledge, this is the first study to assess adherence rates of a single pharmaceutical agent across different medical conditions in a consistent, unified manner.

Published estimates of medication adherence in the United States vary across different medical conditions, but it is thought that adherence among patients with chronic conditions is lower than among those with acute conditions. ${ }^{27}$ Indeed, as few as $50 \%$ of patients with chronic conditions take their medications as prescribed. ${ }^{28}$ Overall, the duloxetine adherence rates that we observed were consistent with these findings. In none of the six conditions we studied did duloxetine adherence rates exceed $40 \%$. Across all studied conditions, the adherence rate for duloxetine averaged $34.6 \%$ (mean MPR $=0.54$ ).

We found modest variation in adherence rates across the six medical conditions included in this study. These variations were most prominent in comparisons of mental health versus chronic pain conditions. Adherence rates were
Table 4 Odds ratios and $95 \% \mathrm{Cls}$ for stepwise logistic regression of adherence (part 2)

\begin{tabular}{|c|c|c|}
\hline & \multicolumn{2}{|l|}{ Adherence } \\
\hline & Odds ratio & $95 \% \mathrm{Cl}$ \\
\hline \multicolumn{3}{|l|}{ Body system disorder } \\
\hline Infection & 0.89 & $0.82-0.96$ \\
\hline Nerve & 1.10 & $1.03-1.17$ \\
\hline \multicolumn{3}{|l|}{ Comorbid health conditions } \\
\hline Dyslipidemia & 1.14 & $1.07-1.22$ \\
\hline Insomnia & 0.88 & $0.78-0.99$ \\
\hline Osteoarthritis (prior to look-back) & 1.14 & $1.02-1.26$ \\
\hline Miscellaneous pain & 0.88 & $0.83-0.94$ \\
\hline Psychiatric disorders $^{\mathrm{a}}$ & 0.83 & $0.76-0.91$ \\
\hline Sleep apnea & 1.21 & $1.06-1.38$ \\
\hline \multicolumn{3}{|l|}{ Prior medication use } \\
\hline Benzodiazepines & 0.84 & $0.79-0.90$ \\
\hline Duloxetine (pre-washout) & 0.57 & $0.51-0.65$ \\
\hline Muscle relaxants & 0.92 & $0.85-0.98$ \\
\hline Opioids & 0.74 & $0.60-0.92$ \\
\hline SNRIs (nonduloxetine) & 1.34 & I.22-1.47 \\
\hline SSRIs & 1.15 & $1.08-1.22$ \\
\hline
\end{tabular}

Note: aRefers to psychiatric disorders other than major depressive disorder or generalized anxiety disorder.

Abbreviations: $\mathrm{Cl}$, confidence interval; SNRI, selective norepinephrine reuptake inhibitor; SSRI, selective serotonin reuptake inhibitor.

highest among patients with MDD (37.3\%). The lowest rates of duloxetine adherence were recorded among patients with CLBP $(29.9 \%)$ and only slightly higher among those with DPNP (31.0\%). Adherence rates were statistically significantly greater among patients with MDD than among any other cohort, save the OA cohort, while significantly lower among patients with CLBP than among any other cohort, other than the DPNP cohort. Averaged together, the adherence rate for MDD and GAD was $36.7 \%$, while for the four chronic pain conditions, it was $32.6 \%(P<0.0001)$.

Our estimate of duloxetine adherence rates among patients with MDD was similar to that reported by Liu et al ${ }^{18}$ (38.1\%), but much different than that reported by Liu et $\mathrm{al}^{6}$ (55.8\%). Much of the difference between these estimates is most likely due to the use of a 180 -day versus 365 -day adherence rate measure, reinforcing the importance of using consistent methodologies to compare measures of adherence across different studies.

Results of the stepwise logistic regression were very similar to those reported in individual MDD, FM, and DPNP studies. ${ }^{4-6,17-20}$ The dichotomy between mental and chronic pain was further supported by results of our logistic regression analysis. After adjustment for potentially confounding factors, the odds of duloxetine adherence among patients with GAD did not differ significantly from those with MDD, despite a statistically significant difference in the observed 
rates. The lower adherence rates among patients with GAD may be caused by higher percentages of younger male patients in that cohort since our regression analysis revealed that lower adherence rates are associated with these demographic groups. Conversely, the odds of adherent behavior among patients with OA were significantly lower than for the MDD cohort, despite the fact that the observed rates for OA and MDD were not significantly different. This disparity between observed and calculated rates may be caused by higher percentages of older, female patients in the OA cohort. No significant differences in the odds of adherence across the CLBP, OA, and DPNP patient cohorts were noted.

As for the differences observed across the mental and physical pain spaces, our regression analysis identified that prior use of SSRI antidepressants had a positive impact on adherence, and prior use of pain medications, particularly opioids, had a negative impact. Patients having previously experienced SSRI treatment, who are more commonly found among depressed or anxious patients than among patients with chronic pain (without comorbid depression), may have better tolerance and response than those without such experiences. Duloxetine inhibits both serotonin and norepinephrine reuptake. If patients have used SSRIs in the past, the risks for some side effects and intolerability due to serotonin reuptake inhibition might be reduced. ${ }^{17}$ On the other hand, prior use of pain medications, and in particular, opioids, is a more common occurrence in the chronic pain- than the mental health-related cohorts, and this may promote certain patients' beliefs about their condition that are different from patients without such experiences. Patients with a history of prior pain medications may have more experience with "as needed" treatment regimens, which may make deviating from recommended daily dosing of duloxetine more common. Similarly, patients with prior pain medication experience, particularly those with a history of opioid medications, may have concerns with the potential for addiction to any medication used for pain, despite duloxetine's nonaddictive profile. They may, therefore, fail to follow completely their physician's recommendations regarding dosage amount and frequency, thereby reducing their calculated medication adherence rates. Other differences between conditions may have to do with differences in efficacy, for which information is unavailable in claims data sets.

Several limitations associated with this study are worthy of consideration. Primary among these is, as with all studies of this type, that we based the calculation of MPR and length of therapy on pharmacy claims data. Ideally, these calculations would be based on drug exposure rather than drug acquisition. However, research has shown that the rate of drug acquisition is predictive of drug exposure. ${ }^{29}$ Another limitation is that pharmacy claims records, such as those used in this study, do not indicate directly the medical condition for which a drug is prescribed, so the accuracy of our data depends on the correlation of pharmacy and medical claims within prespecified data ranges. Further, the integrated claims data set used in this analysis does not include information on benefit structure and, hence, is incapable of providing any information regarding possible differences in plan structure across diseases. Also, our analyses of adherence in the treatment of CLBP and OA predated the United States Food and Drug Administration's approval of duloxetine for use in these conditions. Findings for CLBP and OA are thus to be viewed as providing early evidence for adherence rates in these two chronic musculoskeletal pain conditions, which are to be revisited as more data become available. Also, the analyses conducted were limited to patient cohorts associated with only one of the six targeted medical conditions. Analysis of the impact of comorbidities across these disease states was beyond the scope of this study. Finally, the primary objective of this study was to use a standardized set of assumptions for calculating adherence rates and, therefore, our measures were based, of necessity, on arbitrary cutoffs. For example, we defined adherence based on 365 days. To determine the impact of this definition, we also calculated adherences based on 180 days and 90 days. Adherence rates based on these cutoffs were larger, but the relationships between these rates across cohorts remained the same (data not shown). Similarly, changing the cutoff gap between prescriptions from 30 days to 15 days or 45 days did not change the relative adherence rates across the studied conditions (data not shown).

\section{Conclusion}

Treatment adherence among patients who were newly initiated on duloxetine varied modestly across the medical conditions for which it was used. After adjusting for potential confounders, the differences between the studied mental conditions (MDD and GAD), and three of the four chronic pain conditions, were clearly established. These results suggest that not just the drug, but the particular condition treated, should be taken into account by health plans when measuring, monitoring, and comparing adherence rates.

\section{Acknowledgments}

This research was funded by Eli Lilly and Company, Indianapolis, IN, USA. The authors would like to thank Rodney J Moore, PhD (inVentiv Health Clinical, funded 
by Eli Lilly and Company) for his assistance in drafting the manuscript.

\section{Disclosure}

Drs Able, Cui, and Shen are employees and minor stockholders of Eli Lilly and Company. The authors report no other conflicts of interest in this work.

\section{References}

1. Melfi CA, Chawla AJ, Croghan TW, Hanna MP, Kennedy S, Sredl K. The effects of adherence to antidepressant treatment guidelines on relapse and recurrence of depression. Arch Gen Psychiatry. 1998;55(12):1128-1132.

2. Hughes DA, Bagust A, Haycox A, Walley T. The impact of noncompliance on the cost-effectiveness of pharmaceuticals: a review of the literature. Health Econ. 2001;10(7):601-615.

3. Sokol MC, McGuigan KA, Verbrugge RR, Epstein RS. Impact of medication adherence on hospitalization risk and healthcare cost. Med Care. 2005;43(6):521-530.

4. Wu N, Chen S, Boulanger L, Fraser K, Bledsoe SL, Zhao Y. Duloxetine compliance and its association with healthcare costs among patients with diabetic peripheral neuropathic pain. J Med Econ. 2009;12(3):192-202.

5. Wu N, Chen S, Boulanger L, Rao P, Zhao Y. Average daily dose, medication adherence, and healthcare costs among commerciallyinsured patients with fibromyalgia treated with duloxetine. Curr Med Res Opin. 2011;27(6):1131-1139.

6. Liu X, Tepper PG, Able SL. Adherence and persistence with duloxetine and hospital utilization in patients with major depressive disorder. Int Clin Psychopharmacol. 2011;26(3):173-180.

7. Fleming WK. Pharmacy management strategies for improving drug adherence. J Manag Care Pharm. 2008;14(6 Suppl B):16-20.

8. Yeaw J, Benner JS, Walt JG, Sian S, Smith DB. Comparing adherence and persistence across 6 chronic medication classes. J Manag Care Pharm. 2009;15(9):728-740.

9. Briesacher BA, Andrade SE, Fouayzi H, Chan KA. Comparison of drug adherence rates among patients with seven different medical conditions. Pharmacotherapy. 2008;28(4):437-443.

10. Bymaster FP, Dreshfield-Ahmad LJ, Threlkeld PG, et al. Comparative affinity of duloxetine and venlafaxine for serotonin and norepinephrine transporters in vitro and in vivo, human serotonin receptor subtypes, and other neuronal receptors. Neuropsychopharmacology. 2001;25(6):871-880.

11. Detke MJ, Lu Y, Goldstein DJ, Hayes JR, Demitrack MA. Duloxetine, $60 \mathrm{mg}$ once daily, for major depressive disorder: a randomized double-blind placebo-controlled trial. J Clin Psychiatry. 2002;63(4): 308-315.

12. Norman TR, Olver JS. Duloxetine in the treatment of generalized anxiety disorder. Neuropsychiatr Dis Treat. 2008;4(6):1169-1180.

13. Chappell AS, Bradley LA, Wiltse C, Detke MJ, D'Souza DN, Spaeth M. A six-month double-blind, placebo-controlled, randomized clinical trial of duloxetine for the treatment of fibromyalgia. Int J Gen Med. 2008;1:91-102.
14. Smith T, Nicholson RA. Review of duloxetine in the management of diabetic peripheral neuropathic pain. Vasc Health Risk Manag. 2007;3(6):833-844.

15. Chappell AS, Desaiah D, Liu-Seifert H, et al. A double-blind, randomized, placebo-controlled study of the efficacy and safety of duloxetine for the treatment of chronic pain due to osteoarthritis of the knee. Pain Pract. 2011;11(1):33-41.

16. Skljarevski V, Zhang S, Desaiah D, et al. Duloxetine versus placebo in patients with chronic low back pain: a 12-week, fixed-dose, randomized, double-blind trial. J Pain. 2010;11(12):1282-1290.

17. Gelwicks S, Faries DE, Liu X. Predictors of duloxetine treatment persistence for patients with major depressive disorder. Health Outcomes Research in Medicine. 2011;2:e3-e13.

18. Liu X, Chen Y, Faries DE. Adherence and persistence with branded antidepressants and generic SSRIs among managed care patients with major depressive disorder. Clinicoecon Outcomes Res. 2011;3:63-72.

19. Cui Z, Zhao Y, Novick D, Faries D. Predictors of duloxetine adherence and persistence in patients with fibromyalgia. J Pain Res. 2012;5: 193-201.

20. Zhao Y, Sun P, Watson P. Medication adherence and healthcare costs among patients with diabetic peripheral neuropathic pain initiating duloxetine versus pregabalin. Curr Med Res Opin. 2011;27(4):785-792.

21. Ivanova JI, Birnbaum HG, Kantor E, Schiller M, Swindle RW. Duloxetine use in chronic low back pain: treatment patterns and costs. Pharmacoeconomics. 2012;30(7):595-609.

22. Andrade SE, Kahler KH, Frech F, Chan KA. Methods for evaluation of medication adherence and persistence using automated databases. Pharmacoepidemiol Drug Saf. 2006;15(8):565-574; discussion 575-577.

23. Cramer JA, Roy A, Burrell A, et al. Medication compliance and persistence: terminology and definitions. Value Health. 2008;11(1):44- 47.

24. Karve S, Cleves MA, Helm M, Hudson TJ, West DS, Martin BC. Prospective validation of eight different adherence measures for use with administrative claims data among patients with schizophrenia. Value Health. 2009;12(6):989-995.

25. Cantrell CR, Eaddy MT, Shah MB, Regan TS, Sokol MC. Methods for evaluating patient adherence to antidepressant therapy: a realworld comparison of adherence and economic outcomes. Med Care. 2006;44(4):300-303.

26. Cymbalta (duloxetine delayed-release capsules) [package insert]. Indianapolis, IN: Eli Lilly and Company; 2004 [revised November 9, 2012]. Available from: http://pi.lilly.com/us/cymbalta-pi.pdf. Accessed July 10, 2013.

27. Osterberg L, Blaschke T. Adherence to medication. $N$ Engl J Med. 2005;353(5):487-497.

28. Santamarina ML, DiMaggio EC. Prescription drug adherence: A "challenge" for so many ... Plainsboro, NJ: Pharmacy Times [serial on the Internet]; Dec 2009. Available from: http://www.pharmacytimes. com/print.php?url=/publications/issue/2009/December2009/ P2PAdherence-1209. Accessed September 18, 2013.

29. Choo PW, Rand CS, Inui TS, et al. Validation of patient reports, automated pharmacy records, and pill counts with electronic monitoring of adherence to antihypertensive therapy. Med Care. 1999;37(9): 846-857.
ClinicoEconomics and Outcomes Research

\section{Publish your work in this journal}

ClinicoEconomics \& Outcomes Research is an international, peerreviewed open-access journal focusing on Health Technology Assessment, Pharmacoeconomics and Outcomes Research in the areas of diagnosis, medical devices, and clinical, surgical and pharmacological intervention. The economic impact of health policy and health systems

\section{Dovepress}

organization also constitute important areas of coverage. The manuscript management system is completely online and includes a very quick and fair peer-review system, which is all easy to use. Visit http://www.dovepress.com/testimonials.php to read real quotes from published authors. 